\title{
Segregation mechanisms of tissue cells: from experimental data to models
}

\author{
Előd Méhes ${ }^{1 *}$ and Tamás Vicsek $k^{1,2^{*}}$
}

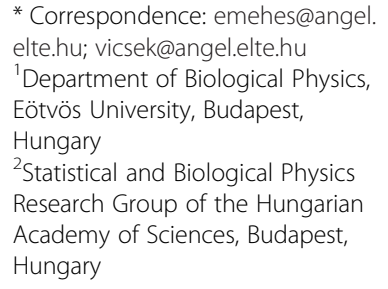

* Correspondence: emehes@angel. elte.hu; vicsek@angel.elte.hu ${ }^{1}$ Department of Biological Physics, Eötvös University, Budapest, Hungary

${ }^{2}$ Statistical and Biological Physics Research Group of the Hungarian Academy of Sciences, Budapest, Hungary

\begin{abstract}
Considerable advance has been made in recent years in the research field of pattern formation by segregation of tissue cells. Research has become more quantitative partly due to more in-depth analysis of experimental data and the emergence modeling approaches. In this review we present experimental observations, including some of our new results, on various aspects of two and three dimensional segregation events and then summarize the computational modeling approaches.
\end{abstract}

Keywords: Segregation, Cell, Culture, Sorting, Motility, Differential adhesion, Development, Model, Simulation

\section{Review}

Introduction

Pattern formation by concerted motion of large populations of different cell types is a ubiquitous phenomenon in several biological events, such as embryonic development of various species as well as tumor formation and even with some impact in tissue engineering. Patterns can form as a response by cells to external guidance cues such as morphogens or chemotactic substances or as a process where instead of external cues the local cell-cell interactions and the inherent mechanical or motility characteristics of the cell types will give rise to various multicellular patterns through physical segregation (or sorting) of these cell types. In this review we collect a naturally incomplete list of experimental observations on various aspects of 2- and 3-dimensional segregation events, and then summarize, where available, some computational models on segregation and compare them with experimental findings.

\section{Experimental data on segregation}

\section{Two-dimensional segregation: pattern formation on a substrate}

Basic drives and mechanisms of pattern formation events taking place e.g. during embryonic development can be studied in simplified experimental systems where complexity is reduced and the events are more accessible for quantitative analysis.

In 2-dimensional co-cultures of adherent cells on a rigid substrate Méhes et al. (2012) studied the dynamics of segregation of two initially mixed cell populations into distinct clusters by cell migration in an environment lacking pre-defined external cues. They have found that segregation into large multicellular clusters is facilitated by collective effects in cell motion such as an increase in the directional persistence of

\section{Springer}

(c) 2013 Mehes and Vicsek; licensee Springer. This is an Open Access article distributed under the terms of the Creative Commons Attribution License (http://creativecommons.org/licenses/by/2.0), which permits unrestricted use, distribution, and reproduction in any medium, provided the original work is properly cited. 
constituent cells. The growth of such multicellular clusters by consecutive fusion of smaller clusters follows algebraic scaling law with characteristic exponents depending on the collective effects (Figure 1 also see Additional file 1). The growth exponent values measured in this cell culture system with self-propelled collective motion exceed the exponent values resulting from computer simulations with diffusively moving segregating units detailed in (Nakajima \& Ishihara 2011).

Inducing directional migration in the form of migration down a cell density gradient during the segregation of initially mixed co-cultures had no considerable impact on the dynamics of segregation. Clusters of similar size formed with similar dynamics as compared to mixed co-cultures with no cell density gradient (Figure 2).

\section{Segregation events in three dimensions: gastrulation and tissue organization}

Three-dimensional segregation of cell populations is most prominent during gastrulation, the early phase of embryonic development resulting in the formation of main germ layers that later on give rise to all tissues. Gastrulation is a spectacular event under the microscope involving collective motion of large number of cells, but although gastrulation events have been known since early embryonic works at the beginning of the $20^{\text {th }}$ century, the basic mechanisms that provide for both its accuracy and robustness are just being uncovered. Segregation of cell populations adopting different cell fates into distinct domains is governed by their mechanical properties and active motion, and constitutes an important driving mechanism of gastrulation and tissue organization. Segregation is also important in other embryonic processes, such as
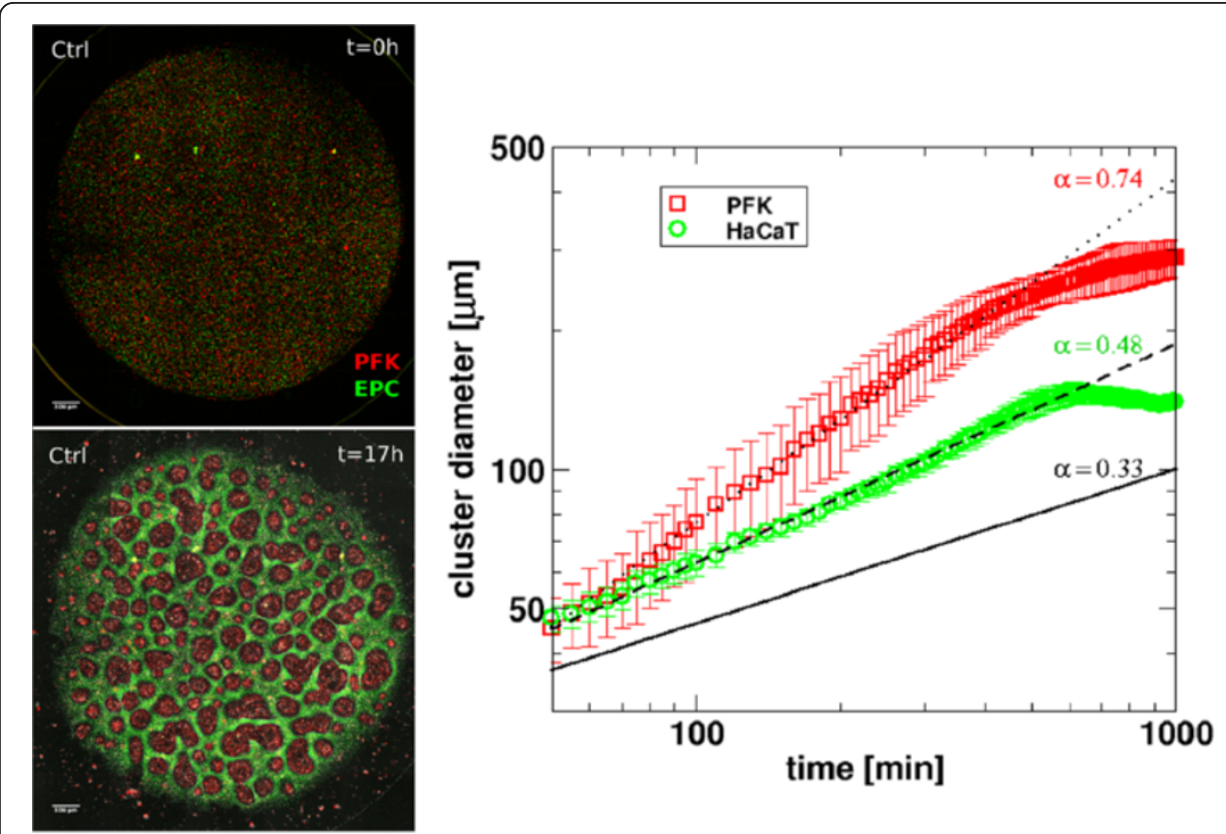

Figure 1 Dynamics of 2-dimensional segregation of keratocytes in culture. Left: Segregation in mixed cocultures of primary goldfish keratocytes (PFK, red) and EPC fish keratocytes (EPC, green), consisting of $>250000$ cells. Upper panel shows initial stage after cell attachment, lower panel shows final stage after 17 hours of cell migration. Scale bar is $100 \mu \mathrm{m}$. An additional movie file shows the dynamics of this in more detail [see Additional file 1]. Right: Average cluster diameter growth curves calculated from experiments with primary goldfish keratocytes (PFK) or human keratocytes (HaCaT). Exponent values obtained from fitting straight line segments to experimental curves are shown. Cluster growth curve of simulated segregation of cells without collective motion characterized by exponent value $a=0.33$ is shown for reference (black solid line). From Mehes et al. (2012). 


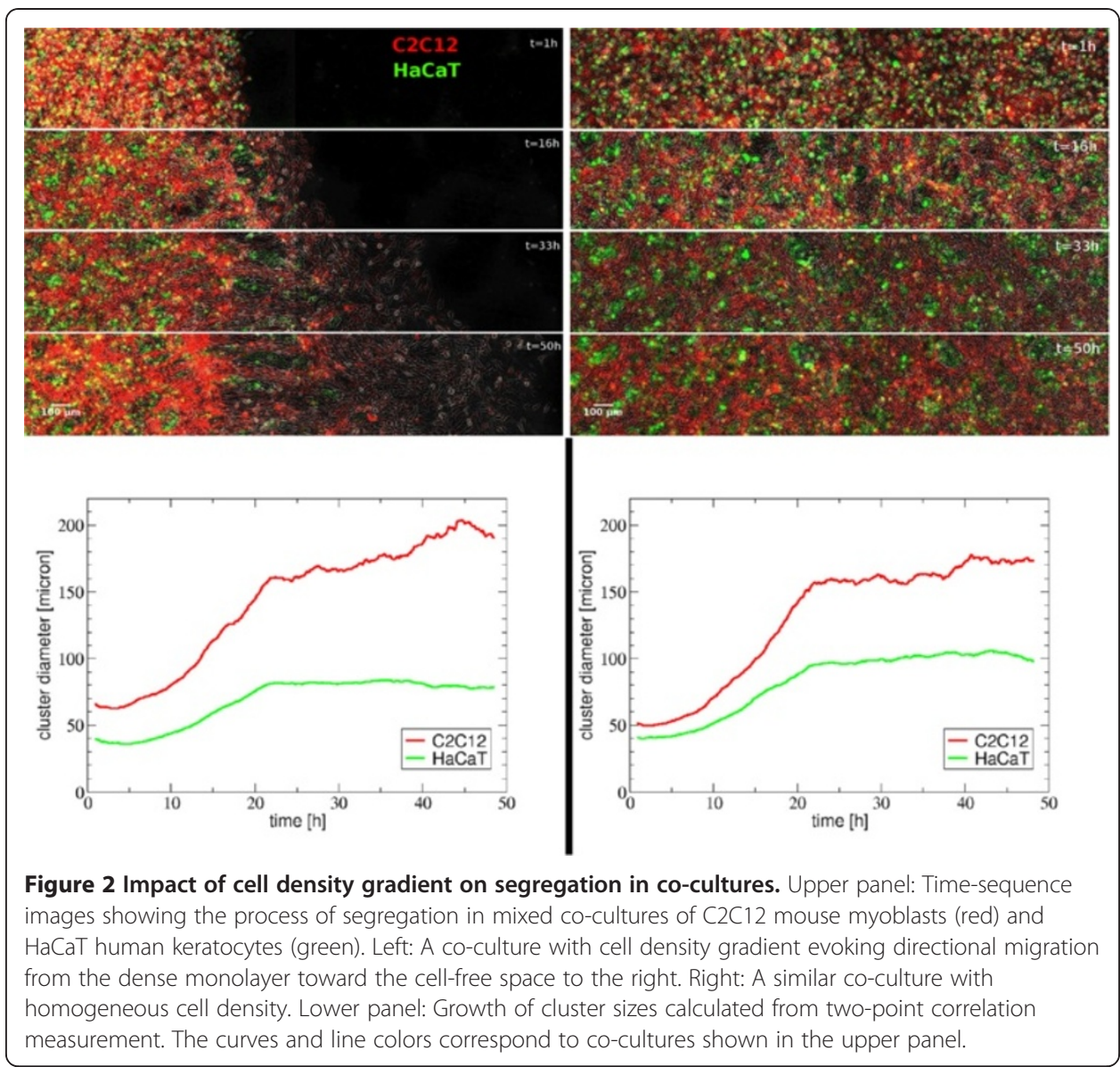

blastocyst formation in mammals or the development of the initial blood vessel network in birds, where precursor cells differentiate in a spatially random pattern and they have to find their way to join and form an organ.

Cell segregation was first demonstrated by the experiments of Townes and Holtfreter in which presumptive neural and epidermal cells were isolated from amphibian gastrulas, subsequently they were mixed and they autonomously sorted into separate tissues (Townes \& Holtfreter 1955). In similar early experiments, mixed cells isolated from the adult Hydra were shown to segregate and form separate tissues (Gierer et al. 1972).

Segregation of various cell types in 3-dimensions was studied is several works (Foty et al. 1996; Beysens et al. 2000; Foty \& Steinberg 2005; Krieg et al. 2008; Schötz et al. 2008), aiming to explain the observed configurations of segregated domains, typically the envelopment of one cell type by the other, evolving from an initial mixture of cells. These in vitro segregating systems are considered to be analogous to non-mixing liquids and their segregation is shown to be driven by differences in tissue surface tension (TST) of the constituent cell types (Foty et al. 1996). Several works tested the contribution of cell-cell adhesion (Foty \& Steinberg 2005; Schötz et al. 2008) and cell cortex tension (Krieg et al. 2008; Maître \& Heisenberg 2011) to TST. The developmental aspects of cell sorting and TST are detailed in a recent review by Krens and Heisenberg (2011). 
Experimental proof on the relative weights of adhesion and cell cortex tension in controlling cell-cell contact formation in zebrafish germ layer progenitors and determining the experimentally measurable separation force between cell pairs was provided by Maître et al. (2012). Cells are described as fluid objects with viscoelastic cortex under tension and adhesive bonds maintaining cell-cell contacts. Contact expansion is controlled by cell cortex tension at the contact, generated by myosin-2 activity, while adhesion by cadherins (membrane-spanning adhesion molecules) mechanically couples the adhering cells, and such coupling is limited by cadherin anchorage to the sub-membrane cortex. Cell segregation is mainly driven by contact formation by the active reduction of cell cortex tension at cell-cell interface, which leads to decrease in cell-cell interface tension, while cell cortex tension at the cell-medium interface is maintained, accounting for maintained TST. Adhesion is shown to have little direct function in contact expansion. Considering the typical cadherin density, the adhesion energy per unit area of the cell surface $\left(\sim 1 \times 10^{-7} \mathrm{~N} / \mathrm{m}\right)$ is several orders of magnitude lower than typical TST measured in cell aggregates (being in the order of $1 \times 10^{-3} \mathrm{~N} / \mathrm{m}$ ) (Amack \& Manning 2012).

\section{Emerging hypotheses}

Two opposing hypotheses have been developed for explaining the origin of tissue surface tension. One is the differential adhesion hypothesis (DAH), developed by M. Steinberg (1962a); (1962b); Steinberg 1963; Steinberg 1996) postulating that tissue surface tension is proportional to the intensity of adhesive energy between point object cells. This hypothesis was elaborated in extensive modeling approaches by J. Glazier (1992). Experimental studies showed that TST is proportional to cadherin levels (Foty \& Steinberg 2005).

The other hypothesis is the differential interfacial tension hypothesis (DITH), developed by Harris (1976), Brodland (2002; Brodland 2003) and Graner (1993), postulating that tissue surface tension arises from cortical tension of individual cells generated by actomyosin contractility, while a cell's mechanical energy changes with cell shape. This model was also supported by experimental data on cell cortex tension and TST (Krieg et al. 2008).

A model integrating cell-cell adhesion and contractility of cell interfaces in the generation of tissue surface tension, the driving force of cell segregation and tissue spreading, was provided by Manning et al. (2010). This model specifies an explicit relationship between surface tension and the ratio of effective adhesion $(\gamma)$ to cortical tension $(\beta)$. Effective adhesion, $\gamma$, is the net energetic contribution of contacting cell surfaces, which depends on the free energy of cadherin bonds and local changes in cell cortex tension. Surface tension exhibits a crossover at $\gamma / \beta \sim 2$ from adhesion-dominated behavior (DAH) in the regime of $\gamma / \beta<2$ to a dependence on cortical tension and other mechanical effects in the regime of $\gamma / \beta>2$.

A recent review emphasizes the role of boundary cells in TST as they can actively change their mechanical properties generating different cortical tensions along their internal and external interfaces. Such 'mechanical polarization' is suggested to exert the same net mechanical effect on the tissue as if extra adhesion was introduced among all cells and it is hypothesized to dominate TST instead of the mechanical energy of adhesive bonds (Amack \& Manning 2012). Strong apical-basal actin polarization was indeed shown in surface cells in zebrafish embryonic explants (Krens et al. 2011). Considering the low adhesion energy of cadherins, the findings that TST is proportional to the 
number of surface cadherins (Foty \& Steinberg 2005) can also be interpreted in a way that it is signaling through more cadherins leading to increased actomyosin contractility and resultant cell cortex tension which generates higher TST.

\section{Experimental observations}

The dynamics of growth of segregated domain size in 3-dimensions was studied by Beysens et al., (2000) using mixed cultures of embryonic pigmented epithelial cells and neural retinal cells, which segregated and formed enveloped structure over time in a configuration determined by surface tensions of the cell types. As a comparison, the segregation of gas and liquid phases was studied under microgravity resulting in similar segregated configuration determined by surface tension (Figure 3). Authors have found a linear increase in the size of segregated cell domains in time.

In a study quantifying the adhesive and mechanical properties of zebrafish germ line progenitor cell types Krieg et al. (2008) investigated the role of tensile forces in cell segregation. Using single-cell force spectroscopy they have measured the cell-cortex tension of these cell types (ectoderm, mesoderm and endoderm) while specifically interfering with actomyosin-dependent cell-cortex tension. Performing segregation experiments using cell types with altered myosin activity they have demonstrated that differential actomyosindependent cell-cortex tension is required and sufficient to direct the segregation of cell types and determine the final configuration of the segregated domains.

The dynamics of 3-dimensional segregation of mixed germ line progenitors of the zebrafish was studied by Klopper et al. (2010). As segregation proceeds in this system, the domain consisting of mesoderm cells gradually engulfs the ectoderm domain, which
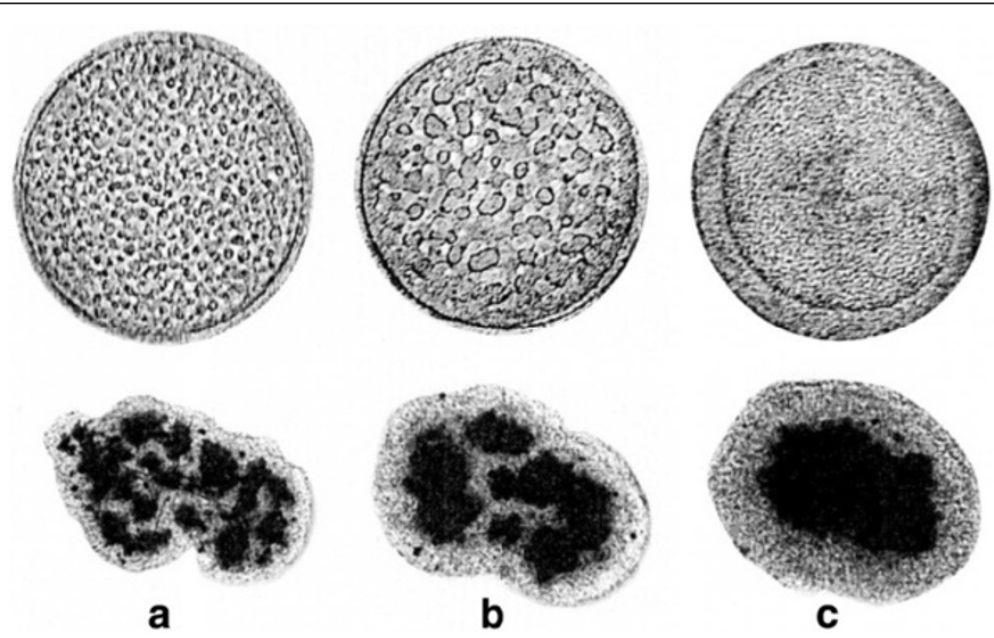

Figure 3 Gas and liquid phase ordering and segregation of retinal cells. Upper panel: Gas and liquid phase ordering in SF6 under reduced gravity, after a thermal quench of $0.7 \mathrm{mK}$ below the critical point (45.564 C). Gas and liquid eventually order with the liquid phase wetting the container wall and surrounding the gas phase, corresponding to wall-liquid interfacial tension $<$ wall-gas interfacial tension. $\mathbf{a}, \mathbf{b}$ and c correspond to 120 s, 275 s and 3960 s after quench, respectively. Lower panel: Sorting out of chicken embryonic pigmented epithelial cells (dark) from chicken embryonic neural retinal cells (light). The average aggregate size is $200 \mu \mathrm{m}$. At the end of sorting, neural retinal cells preferentially wet the external tissue culture medium surrounding the aggregates. Medium-neural retina and medium-pigmented epithelium interfacial tensions are $1.6 \mathrm{dyne} / \mathrm{cm}$ and $12.6 \mathrm{dyne} / \mathrm{cm}$, respectively. $\mathrm{a}, \mathrm{b}$ and c correspond to $17 \mathrm{~h}, 42 \mathrm{~h}$ and $73 \mathrm{~h}$ after initiation of sorting, respectively. From Beysens al. (2000) with permission of Proc. Natl. Acad. Sci. 


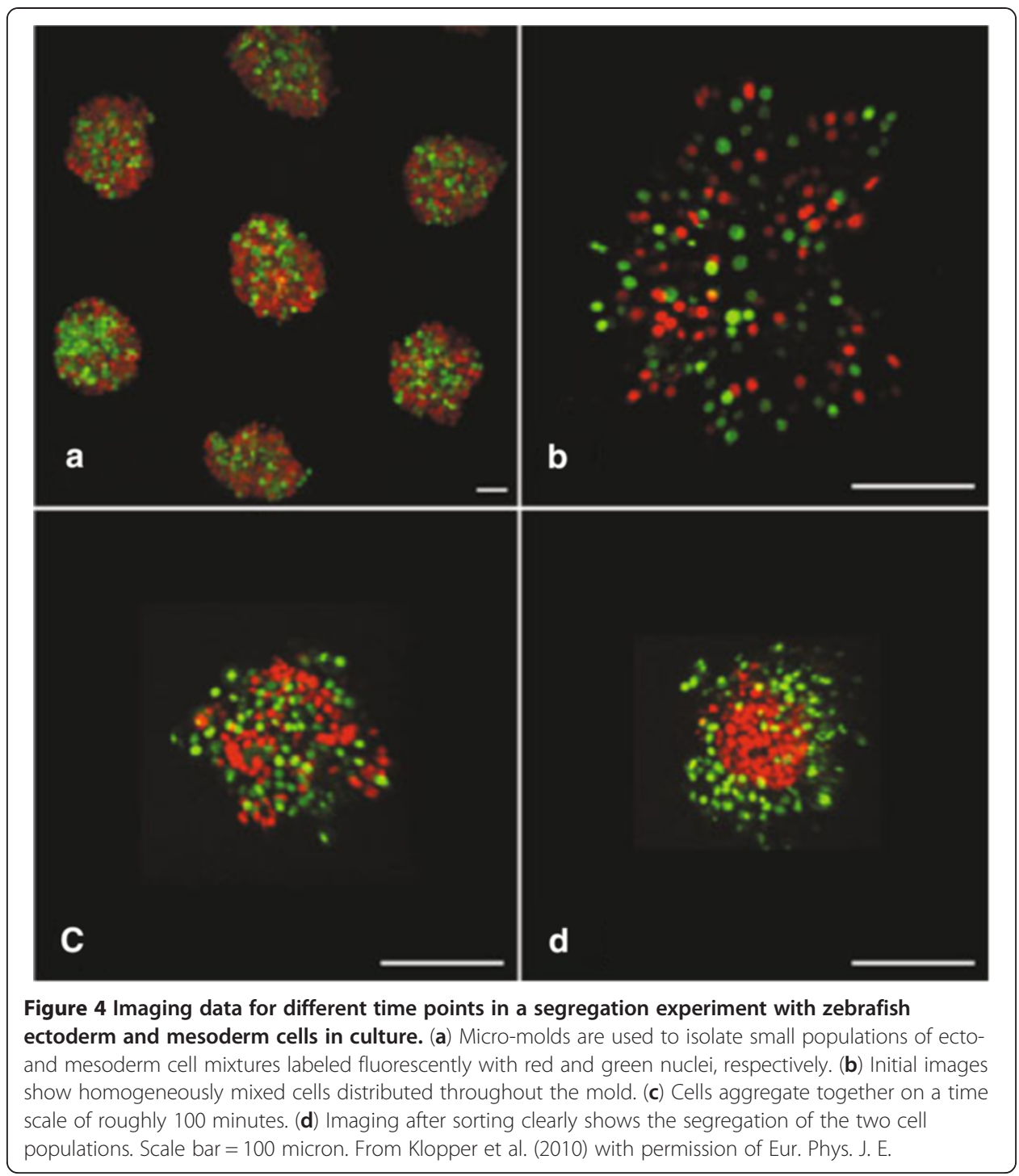

eventually takes the inner position (Figure 4). Authors have monitored the dependence of the local segregation order parameter on system size and found algebraic scaling and different characteristic exponent values for enveloping and engulfed cells.

In a similar in vitro system composed of two mixed epithelial cell types suspended in micromolds, Vicsek and coworkers have recently studied the dynamics of 3-dimensional segregation (manuscript in preparation). The two keratocyte types, primary goldfish keratocytes and EPC fish keratocyte cell line, were labeled with different fluorescent cell tracers and mixed in equal volume ratio. The mixed co-culture was transferred in agarose micromold wells and the ongoing segregation process was imaged by time-lapse videomicroscopy with optical sectioning. Consecutive optical sections were used for reconstructing the evolving 3-dimensional structure of segregated domains as well as analyzing the growth of clusters by two-point correlation method yielding characteristic cluster diameter values. Figure 5 shows snapshots of the evolution of segregation where the forming domains are adjacent and unlike zebrafish germline progenitors there is no engulfment of one domain by the other (see Additional file 2). It was found that the 

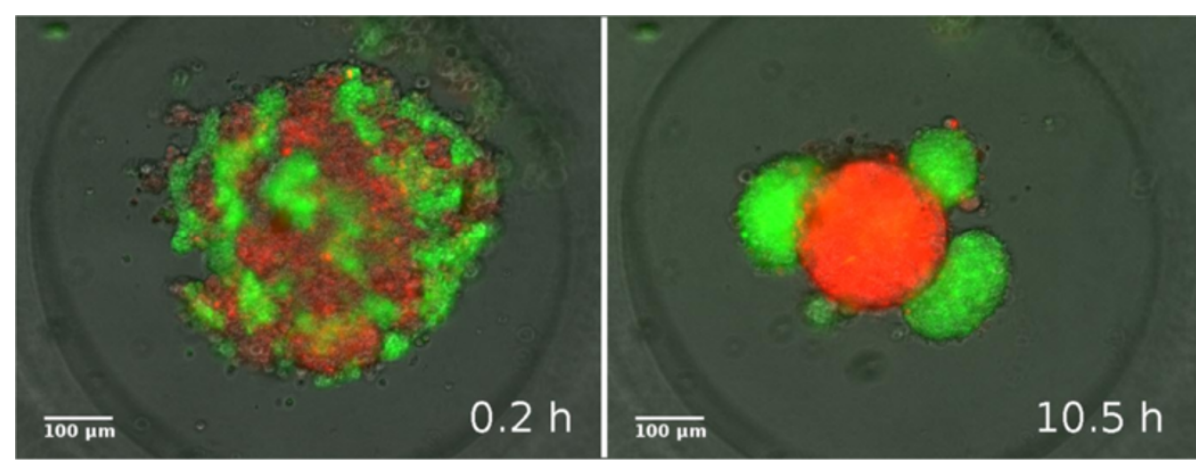

Figure 5 Snapshots from a segregation experiment with two keratocyte types in culture. Left: Initial mixture of primary goldfish keratocytes (red) and EPC fish keratocytes (green) in a micro-mold. Right: Homotypic cell clusters formed through segregation. An additional movie file shows this in more detail [see Additional file 2].

growth of segregated domain size follows algebraic scaling law and it is fast, typically completed within 6 hours. These observations are in contrast with earlier simulations (Belmonte et al. 2008) suggesting a much slower process (discussed in the section "Segregation by collective motion and adhesion").

In early embryonic development, during the blastocyst formation in mice, cell fate specification of epiblast and primitive endoderm cells occurs in a spatially mixed way, followed by their active segregation into distinct domains (Morris et al. 2010). Experimental studies of this process have revealed that segregation cannot be attributed to differential adhesion only but other processes such as active motion and apicalbasolateral polarization of surface endoderm cells eventually surrounding the epiblast cells appear to contribute to it (Moore et al. 2009). It is also proposed and that repositioning of cells in turn influences their fate.

Pattern formation by segregation is a process that is not confined to embryonic development. In a recent publication Inaba et al. (2012) studied the formation of skin pigment patterns in the adult zebrafish. They have demonstrated that segregation of the two pigment cell types eventually forming the stripe pattern is governed by their shortrange repulsive electric interactions that spatially orient their migration.

\section{Models on segregation}

Several computational models exist that attempt to explain and reproduce the experimentally observed segregation processes in various systems. While experimental works tend to focus on the role of tissue surface tension and adhesion in 3-dimensional cell segregation, modeling approaches also include autonomous cell motion and focus on 2 dimensions. A widely accepted model based on the Potts model and the idea of differential cell adhesion was developed by J Glazier and co-workers, later termed as GlazierGraner-Hogeweg model or Cellular Potts Model (Graner \& Glazier 1992). Variants of this model have been successfully employed in simulation works.

\section{Impact of motility on segregation}

Dynamic segregation in 2-dimensions was studied by Kabla (2012) using Cellular Potts Model simulations with self-propelled motile and non-motile cells characterized by 


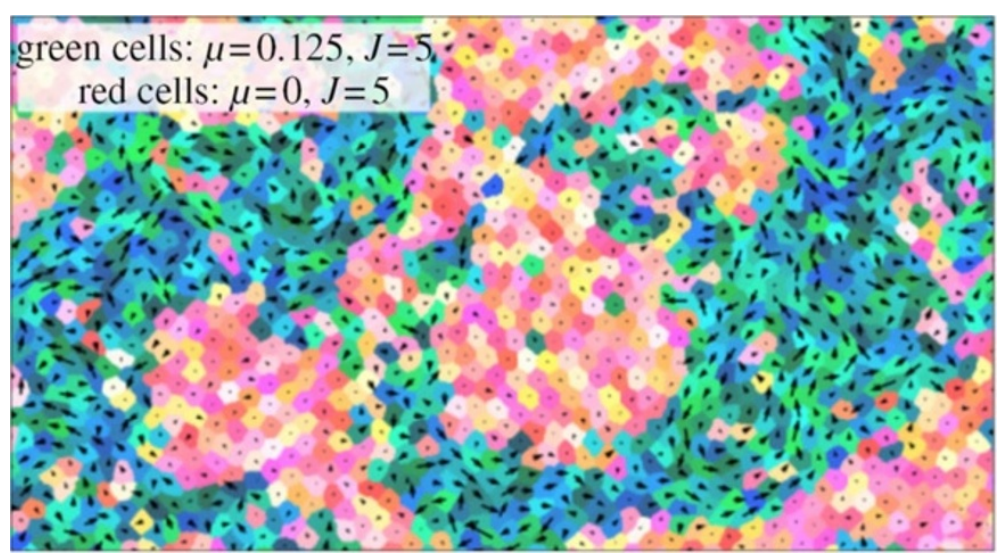

Figure 6 Simulated segregation of motile and non-motile cells. A snapshot of the simulated segregating tissue of motile and non-motile cells at $t=10^{6}$ MCS (Monte-Carlo steps). Membrane tension, $J$, and motile force, $\mu$, of cells are indicated. From Kabla (2012) with permission of J. R. Soc. Interface.

identical adhesive properties. Segregation efficiency has been found to depend on the motile forces controlling cell speed, and efficiency reaches maximum at motile forces close to the threshold required for streaming transition. It is also shown by these simulations that differences in motility are sufficient to drive the segregation of cell populations even without difference in adhesion and as a result motile cells will surround the islands of non-motile cells (Figure 6).

Using Brownian dynamics simulations McCandlish et al. (2011) studied dense mixtures of self-propelled and passive rod-like particles in 2-dimensions where only excluded volume interactions can occur. Adhesion properties do not play a role here, particles only differ in motility. Spontaneous segregation of the two particle species generates a rich array of dynamical domain structures with properties depending on particle shape and propulsion velocity or the combination of these two in the form of particles' Péclet number, a measure similar to the directional persistence of real cells.

Recently, Nakajima et al. (2011) used Cellular Potts Model (CPM) to study the dynamics of the segregation of mixtures of non-self-propelled cell types with diffusive motion. They have found that the increase in the size of segregated domains follows power law and the growth exponent is $n=1 / 3$ for mixtures with 1:1 initial ratio of cell types where segregation proceeds via smoothing of the domain boundary. This is in contrast with previous works with CPM on smaller simulated systems displaying slower logarithmic growth for domain size (Graner \& Glazier 1992; Mombach et al. 1995). CPM simulations with self-propelled cell types characterized by identical adhesive interactions as for the simulations in (Nakajima \& Ishihara 2011) also yield domain growth exponent $\mathrm{n}=1 / 3$ (A. Czirók, personal communication).

\section{Impact of adhesion on segregation}

The role of adhesion in cell segregation was studied by Zhang et al. (2011) using Cellular Potts Model for simulations. In their model they consider variations in the distribution of adhesion molecules per cells. The speed of segregation is found to increase strongly with interfacial tension that depends on the maximum difference in the 


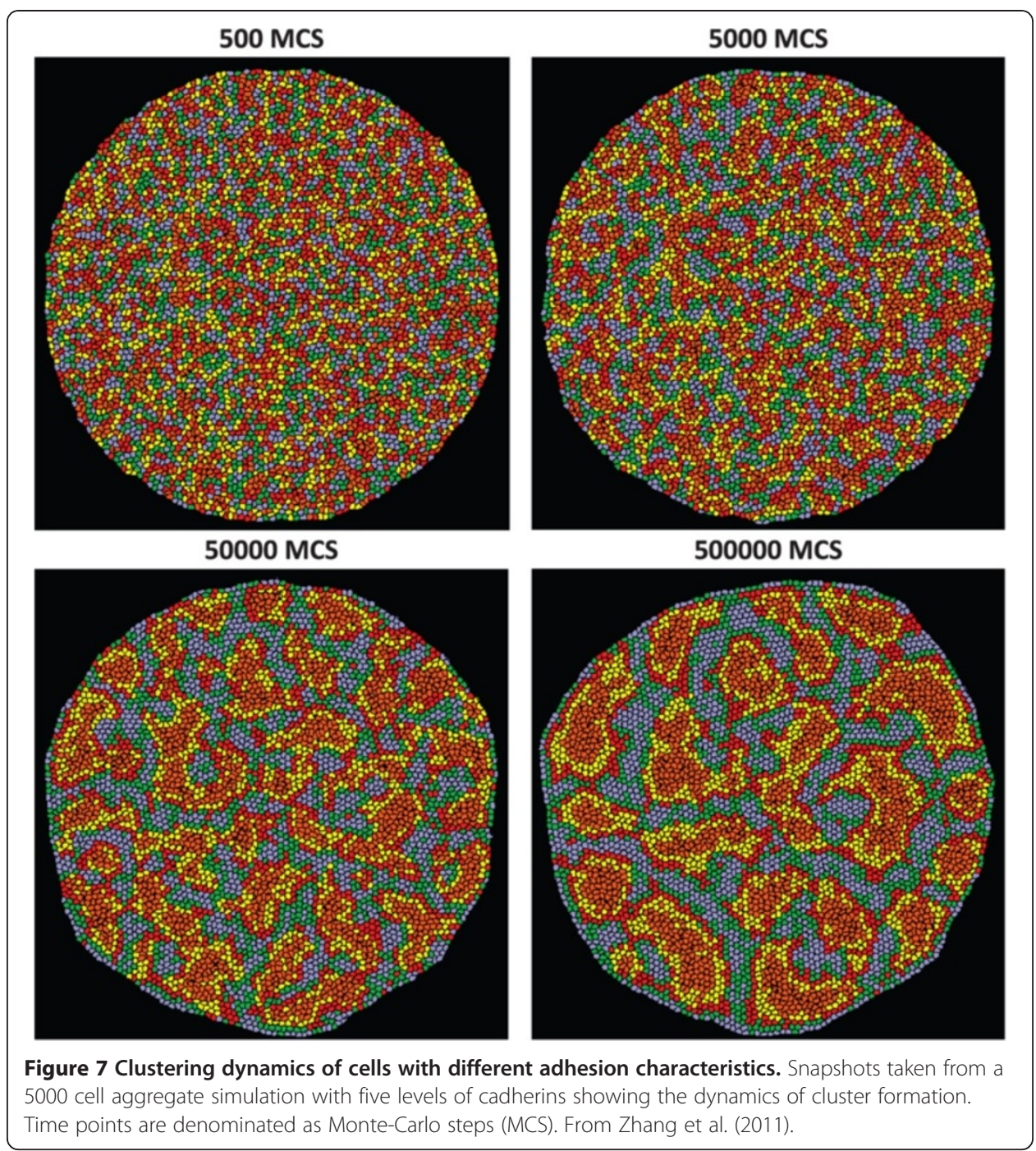

number of cadherin adhesion molecules per cell and the reaction-kinetic models of cadherin binding (Figure 7).

Qualitative description of the dynamical features and the geometry of cell segregation depending on intercellular adhesion parameters is provided by Voss-Böhme et al. (2010) using a stochastic interacting particle model. In this model the hierarchy of segregation is determined by the strengths of adhesive interactions between cells and the boundary.

In a unique paper combining experimental data and modeling Krieg et al. (2008) studied the role of cell-cortex tension and adhesion in segregation. Carrying out simulations using Cellular Potts Model with cell adhesion and cell-cortex tension data derived from experiments they could reproduce the experimentally observed final configurations of segregating germ line progenitor cell types.

\section{Segregation by collective motion and adhesion}

To study cell sorting events Belmonte et al. (2008) developed a model combining the Vicsek Model of collective motion (Vicsek et al. 1995; Vicsek \& Zafeiris 2012) with the 
differential adhesion hypothesis (DAH). In their model $\mathrm{N}$ particles move in 2-dimensional space with constant velocity $v_{0}$. The velocity and the angle of orientation of particle $n$ at time $t$ is denoted by $v_{n}^{t}$ and $\theta_{n}^{t}$, respectively. The new orientation $\theta_{n}^{t+1}$ of particle $n$ is

$$
\theta_{n}^{t+1}=\arg \left[\sum_{m}\left(\alpha_{n m} \frac{\vec{v}_{m}^{t}}{v_{0}}+\beta_{n m} f_{n m}^{t} \vec{e}_{n m}^{t}\right)\right]+\vec{u}_{n}^{t}
$$

where $f_{n m}^{t} e_{n m}^{t}$ is the force exerted by particle $\mathrm{m}$ on particle $\mathrm{n}$ along the direction $e_{n \mathrm{~m}}^{t}$ pointing from particle $\mathrm{m}$ to $\mathrm{n}$. The noise is taken into account by $u_{n}^{t}$ is a unit vector with random, uniformly distributed orientation. $\alpha_{n m}$ and $\beta_{n m}$ are control parameters: $\alpha$ controls the relative weights of the alignment interaction and $\beta$ shows the strength of the radial two-body forces $f_{n m}$, defined as

$$
f_{n m}=\left\{\begin{array}{cllll}
\infty_{r_{n m}} & \text { if } & & r_{n m} \quad<r_{c}, \\
1-\frac{r_{c}}{r_{c}} & \text { if } & r_{c} & <r_{n m}<r_{0}, \\
0 & \text { if } & & r_{n m}>r_{0}
\end{array}\right.
$$

that is for distances smaller than a core radius $r_{c}$ it is a strong repulsive force, around the equilibrium radius $r_{e}$ it is a harmonic-like interaction, whereas for distances larger than the interaction range $r_{O}$ it is set to zero. Having the classic experiments with Hydra cells in mind, authors defined two kinds of particles, "endodermic" and "ectodermic", denoted by 1 and 2, respectively. Accordingly, $\beta_{11}$ and $\beta_{22}$ stand for adhesion within the given cell type, whereas $\beta_{12}=\beta_{21}$ account for symmetric inter-cell-type interactions. Differential adhesion is described by different beta values for symmetric interactions between different cell types. The simulations were performed with cells on a square domain with linear size several magnitudes larger than cell size. Figure 8 shows snapshots from the evolution of the segregation process. Segregation is characterized by an index, $\gamma$, showing the average ratio of dissimilar cells around a cell, for either cell types. This index is decreasing as

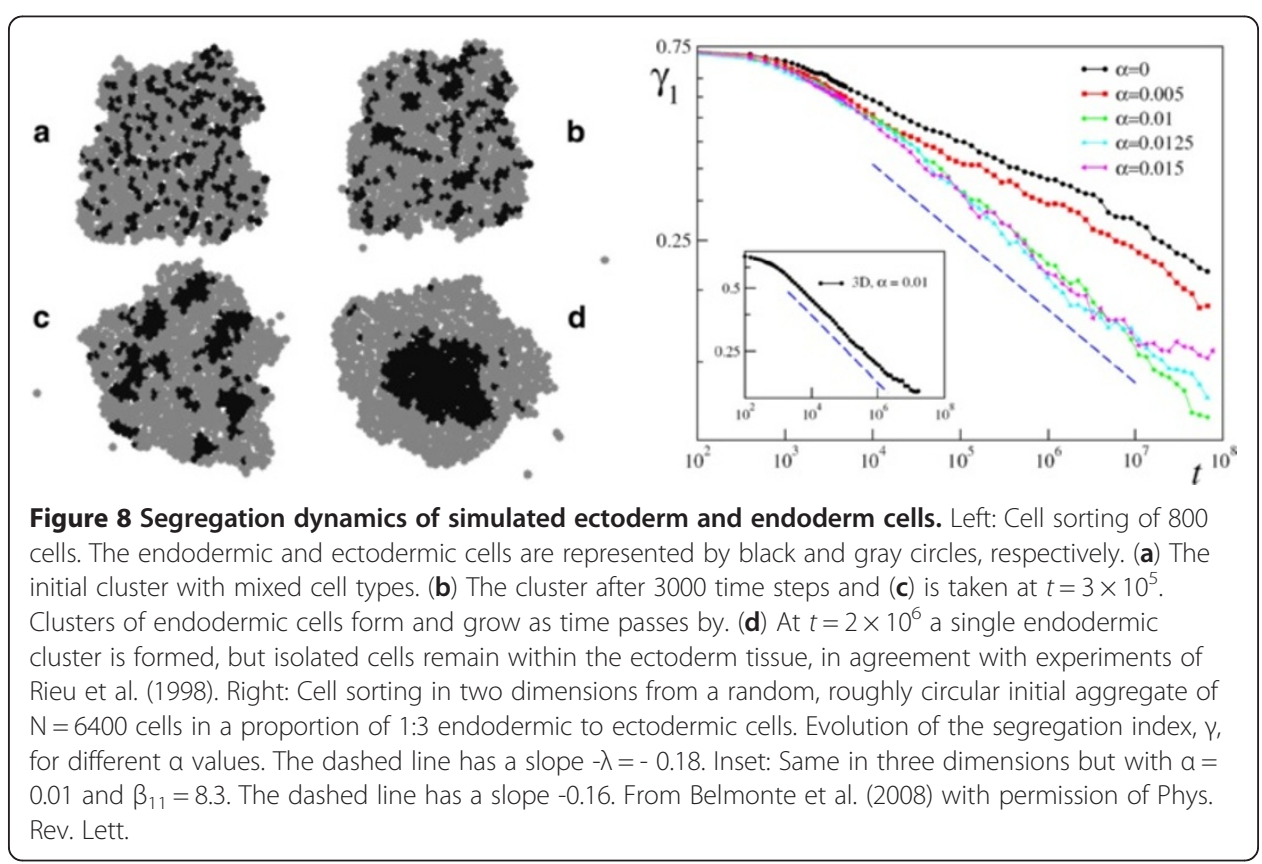


segregation proceeds and it is expected to approach zero in large systems. Authors have found that segregation is characterized by algebraic scaling laws and introducing even a moderate amount of local coherent motion will considerably speed up the segregation process (Figure 8). A variant of this computational model is published by Beatrici et al. (2011) investigating the segregation of self-propelled particles in 2 dimensions, driven by differences only in motility but not in adhesion. In this model, the faster cells envelope the slower cells forming islands as segregation proceeds.

Taking a different approach Honda et al. (2008) studied pattern formation and emergence of asymmetry in mammalian blastocyst formation by simulations using a 3D vertex dynamics model. Endowing the simulated cells with equivalent mechanical properties and assuming that due to basement membrane formation the cell-medium surface in the forming blastocyst cavity is stiffer than cell-cell surfaces, the simulation successfully produced blastocyst structure with variable axis. Enclosing the simulated aggregate in an ellipsoidal capsule corresponding to the zona pellucida, the structure surrounding the blastocyst in vivo, eventually oriented the axis. Authors have shown the autonomous emergence of asymmetry as well as the formation and segregation of distinct cell populations of different sizes in an initially equivalent cell population without preexisting intrinsic differences.

\section{Conclusions}

Our understanding of the events during embryonic development such as multicellular segregation and pattern formation is gradually shifting from a descriptive view towards a causative understanding of the mechanisms. Integrative biological attempts to promote this understanding have been successfully employing various approaches ranging from experimental embryology to statistical physics. Combination of experimental data with multi-disciplinary approaches including computational modeling and statistical physics can help us design more focused experimental tests or predict yet unseen outcomes and these can even further extend our understanding of the dynamic organization of multicellular biological systems.

\section{Additional files}

Additional file 1: mov. 2-dimensional segregation of mixed tissue cells. Segregation in a mixed co-culture of primary goldfish keratocytes (PFK, red) and EPC fish keratocytes (EPC, green) migrating on tissue culture plastic surface is imaged by time-lapse videomicroscopy. Fluorescent cell labels: red: cell tracker CMPTX, green: cell tracker CMFDA. Videomicroscopy duration: 20 hours, images were acquired every 10 minutes by a Zeiss Axio Observer system.

Additional file 2: mov. 3-dimensional segregation of mixed tissue cells. Segregation in a mixed co-culture of primary goldfish keratocytes (PFK, red) and EPC fish keratocytes (EPC, green) suspended in agarose micromold is imaged by time-lapse videomicroscopy. Fluorescent cell labels: red: cell tracker CMPTX, green: cell tracker CMFDA Videomicroscopy duration: 20 hours, images were acquired every 10 minutes by a Zeiss Axio Observer system.

\section{Abbreviations}

DAH: Differential adhesion hypothesis; DITH: Differential interfacial tension hypothesis; TST: Tissue surface tension; CPM: Cellular potts model; PFK: Primary goldfish keratocytes; MCS: Monte-Carlo steps. 
Authors' information

TV is a statistical physicist and he is the principal investigator of the EU ERC COLLMOT project on collective motion. EM is a cell biologist specialized in cell motion research and he is a research fellow in the EU ERC COLLMOT project.

\section{Acknowledgement}

We thank Enys Mones (Dept. Biological Physics, Eötvös University) and András Czirók (Dept. Biological Physics, Eötvös University) for useful consultations. This work was funded by the European Union ERC COLLMOT Project.

Received: 30 November 2012 Accepted: 28 January 2013

Published: 13 March 2013

\section{References}

Amack JD, Manning ML: Knowing the boundaries: extending the differential adhesion hypothesis in embryonic cell sorting. Science 2012, 338:212-215.

Beatrici CP, Brunnet LG: Cell sorting based on motility differences. Phys Rev E Stat Nonlin Soft Matter Phys 2011, 84:031927.

Belmonte JM, Thomas GL, Brunnet LG, de Almeida RM, Chaté H: Self-propelled particle model for cell-sorting phenomena. Phys Rev Lett 2008, 100:248702.

Beysens DA, Forgacs G, Glazier JA: Cell sorting is analogous to phase ordering in fluids. Proc Natl Acad Sci USA 2000, 97:9467-9471.

Brodland GW: The Differential Interfacial Tension Hypothesis (DITH): a comprehensive theory for the selfrearrangement of embryonic cells and tissues. J Biomech Eng 2002, 124:188-197.

Brodland GW: New information from cell aggregate compression tests and its implications for theories of cell sorting. Biorheology 2003, 40:273-277.

Foty RA, Steinberg MS: The differential adhesion hypothesis: a direct evaluation. Dev Biol 2005, 278:255-263.

Foty R, Pfleger CM, Forgacs G, Steinberg MS: Surface tensions of embryonic tissues predict their mutual envelopment behavior. Development 1996, 122:1611-1620.

Gierer A, Berking S, Bode H, David CN, Flick K, Hansmann G, Schaller H, Trenkner E: Regeneration of hydra from reaggregated cells. Nat New Biol 1972, 239:98-101.

Graner F: Can surface adhesion drive cell-rearrangement? J Theor Biol 1993, 164:455-476.

Graner F, Glazier JA: Simulation of biological cell sorting using a two-dimensional extended Potts model. Phys Rev Lett 1992, 69:2013-2016.

Harris AK: Is Cell sorting caused by differences in the work of intercellular adhesion? A critique of the Steinberg hypothesis. J Theor Biol 1976, 61:267-285.

Honda H, Motosugi N, Nagai T, Tanemura M, Hiiragi T: Computer simulation of emerging asymmetry in the mouse blastocyst. Development 2008, 135:1407-1414.

Inaba M, Yamanaka H, Kondo S: Pigment pattern formation by contact-dependent depolarization. Science 2012, 335:677.

Kabla AJ: Collective cell migration: leadership, invasion and segregation. J R Soc Interface 2012, 9:3268-3278.

Klopper AV, Krens G, Grill SW, Heisenberg CP: Finite-size corrections to scaling behavior in sorted cell aggregates. Eur Phys J E Soft Matter 2010, 33:99-103.

Krens SF, Heisenberg CP: Cell sorting in development. Curr Top Dev Biol 2011, 95:189-213.

Krens SF, Möllmert S, Heisenberg CP: Enveloping cell-layer differentiation at the surface of zebrafish germ-layer tissue explants. Proc Natl Acad Sci USA 2011, 108:E9-E10.

Krieg M, Arboleda-Estudillo Y, Puech PH, Käfer J, Graner F, Müller DJ, Heisenberg CP: Tensile forces govern germ-layer organization in zebrafish. Nat Cell Biol 2008, 10:429-436.

Maitre JL, Heisenberg CP: The role of adhesion energy in controlling cell-cell contacts. Curr Opin Cell Biol 2011, 23:508-514

Maitre JL, Berthoumieux H, Krens SF, Salbreux G, Jülicher F, Paluch E, Heisenberg CP: Adhesion functions in cell sorting by mechanically coupling the cortices of adhering cells. Science 2012, 338:253-256.

Manning ML, Foty RA, Steinberg MS, Schoetz EM: Coaction of intercellular adhesion and cortical tension specifies tissue surface tension. Proc Natl Acad Sci U S A 2010, 107:12517-12522.

McCandlish SR, Baskaran A, Hagan MF: Spontaneous segregation of self-propelled particles with different motilities. Soft Matter 2011, 8:2527-2534

Méhes $E$, Mones E, Németh V, Vicsek T: Collective motion of cells mediates segregation and pattern formation in co-cultures. PLoS One 2012, 7:e31711.

Mombach JC, Glazier JA, Raphael RC, Zajac M: Quantitative comparison between differential adhesion models and cell sorting in the presence and absence of fluctuations. Phys Rev Lett 1995, 75:2244-2247.

Moore R, Cai KQ, Escudero DO, Xu XX: Cell adhesive affinity does not dictate primitive endoderm segregation and positioning during murine embryoid body formation. Genesis 2009, 47:579-589.

Morris SA, Teo RT, Li H, Robson P, Glover DM, Zernicka-Goetz M: Origin and formation of the first two distinct cell types of the inner cell mass in the mouse embryo. Proc Natl Acad Sci U S A 2010, 107:6364-6369.

Nakajima A, Ishihara S: Kinetics of the cellular Potts model revisited. New J Phys 2011, 13:033035.

Rieu JP, Kataoka N, Sawada Y: Quantitative analysis of cell motion during sorting in two-dimensional aggregates of dissociated hydra cells. Phys Rev E 1998, 57:924-931.

Schötz E, Burdine RD, Jülicher F, Steinberg MS, Heisenberg CP, Foty RA: Quantitative differences in tissue surface tension influence zebrafish germ layer positioning. HFSP J 2008, 2:42-56

Steinberg MS: On the mechanism of tissue reconstruction by dissociated cells. I. Population kinetics, differential adhesiveness and the absence of directed migration. Proc Natl Acad Sci USA 1962a, 48:1577-1582.

Steinberg MS: Mechanism of tissue reconstruction by dissociated cells. II. Time-course of events. Science 1962b, 137:762-763. 
Steinberg MS: Reconstruction of tissues by dissociated cells. Some morphogenetic tissue movements and the sorting out of embryonic cells may have a common explanation. Science 1963, 141:401-408.

Steinberg MS: Adhesion in development: an historical overview. Dev Biol 1996, 180:377-388.

Townes PL, Holtfreter J: Directed movements and selective adhesion of embryonic amphibian cells. J Exp Zool 1955, 128:53-120

Vicsek T, Zafeiris A: Collective motion. Physics Reports 2012, 517:71-140.

Vicsek T, Czirók A, Ben-Jacob E, Cohen II, Shochet O: Novel type of phase transition in a system of self-driven particles. Phys Rev Lett 1995, 75:1226-1229.

Voss-Böhme A, Deutsch A: The cellular basis of cell sorting kinetics. J Theor Biol 2010, 263:419-436.

Zhang Y, Thomas GL, Swat M, Shirinifard A, Glazier JA: Computer simulations of cell sorting due to differential adhesion. PLoS One 2011, 6:e24999.

doi:10.1186/2194-3206-1-4

Cite this article as: Méhes and Vicsek: Segregation mechanisms of tissue cells: from experimental data to models

Complex Adaptive Systems Modeling 2013 1:4

Submit your manuscript to a SpringerOpen ${ }^{\circ}$ journal and benefit from:

- Convenient online submission

- Rigorous peer review

- Immediate publication on acceptance

- Open access: articles freely available online

- High visibility within the field

- Retaining the copyright to your article

Submit your next manuscript at $>$ springeropen.com 